\section{OPEN ACCESS}

Edited by:

Sabato Santaniello,

University of Connecticut,

United States

Reviewed by:

Antonino Naro,

Centro Neurolesi Bonino Pulejo

(IRCCS), Italy

Tommaso Bocci,

Università degli Studi di Messina, Italy

*Correspondence:

Chunying Liu

liuchunying0302@126.com

Hui Zhang

528zhanghui@sina.cn

Specialty section:

This article was submitted to

Neuroprosthetics,

a section of the journal

Frontiers in Neuroscience

Received: 01 March 2019

Accepted: 11 April 2019

Published: 30 April 2019

Citation:

Guo Y, Bai Y, Xia X, Li J, Wang X,

Dai Y, Dang Y, He J, Liu C and

Zhang H (2019) Effects

of Long-Lasting High-Definition

Transcranial Direct Current

Stimulation in Chronic Disorders of

Consciousness: A Pilot Study.

Front. Neurosci. 13:412.

doi: 10.3389/fnins.2019.00412

\title{
Effects of Long-Lasting
} High-Definition Transcranial Direct Current Stimulation in Chronic Disorders of Consciousness: A Pilot Study

\begin{abstract}
Yongkun Guo ${ }^{1}$, Yang Bai2,3, Xiaoyu Xia ${ }^{4}$, Jinju Li', Xiaoli Wang ${ }^{1}$, Yiwu Dai, Yuanyuan Dang ${ }^{4}$, Jianghong He ${ }^{4}$, Chunying Liu ${ }^{1 *}$ and Hui Zhang ${ }^{1 *}$
\end{abstract}

1 Department of Neurosurgery, Zhengzhou Central Hospital Affiliated to Zhengzhou University, Zhengzhou, China, ${ }^{2}$ International Vegetative State and Consciousness Science Institute, Hangzhou Normal University, Hangzhou, China,

${ }^{3}$ Department of Basic Medical Science, School of Medicine, Hangzhou Normal University, Hangzhou, China,

${ }^{4}$ Department of Neurosurgery, PLA Army General Hospital, Beijing, China

Transcranial direct current stimulation (tDCS) recently was shown to benefit rehabilitation of patients with disorders of consciousness (DOC). However, high-Definition tDCS (HD-tDCS) has not been applied in DOC. In this study, we tried to use HD-tDCS protocol (2 mA, $20 \mathrm{~min}$, the precuneus, and sustaining 14 days) to rehabilitate 11 patients with DOC. Electroencephalography (EEG) and Coma Recovery Scale-Revised (CRS-R) scores were recorded at before (T0), after a single session (T1), after 7 days' (T2), and 14 days' HD-tDCS (T3) to assess the modulation effects. EEG coherence was measured to evaluate functional connectivity during the experiment. It showed that 9 patients' scores increased compared with the baseline. The central-parietal coherence significantly decreased in the delta band in patients with DOC. EEG coherence might be useful for assessing the effect of HD-tDCS in patients with DOC. Long-lasting HD-tDCS over the precuneus is promising for the treatment of patients with DOC.

Keywords: disorders of consciousness, high-definition transcranial direct current stimulation, electroencephalography, Coma Recovery Scale-Revised scores, coherence

\section{INTRODUCTION}

Chronic disorders of consciousness (DOC) consist of vegetative state/ unresponsive wakefulness syndrome (VS/ UWS) and minimally conscious state (MCS) (Giacino et al., 2014). MCS is characterized by minimal but definite behavioral evidence of self or environmental awareness (Giacino et al., 2014). Recently, MCS was subcategorized into MCS- describing low-level behavioral responses and MCS+ describing high level behavioral responses (Bruno et al., 2012). Even though many pharmacological (i.e., amantadine, zolpidem) and non-pharmacological interventions (i.e., deep brain stimulation, spinal cord Stimulation, transcranial magnetic stimulation, median nerve electrical stimulation) have been assessed in the last decade, there remain few effective therapies for patients with DOC (Schiff et al., 2007; Giacino et al., 2012; Della Pepa et al., 2013; Yamamoto et al., 2013; Cossu, 2014; Thibaut et al., 2014; Tucker and Sandhu, 2016). 
Transcranial direct current stimulation (tDCS) is a promising non-invasive brain stimulation technique for treatment of patients with DOC, which is safe, less uncomfortable and easy to handle (Zhang and Song, 2018). tDCS modulates cortical excitability at stimulation sites via weak current which flows through the brain from the anode to the cathode. Anodal tDCS boost neuronal activation via sub-threshold neuronal membrane polarization, and cathodal tDCS can reduce cortical excitability (Lefaucheur et al., 2017). The effects of tDCS stimulation can be long-lasting, and are connected with the duration of stimulation and current intensity (Zhao et al., 2017). Previous studies illustrated the residual capacity for neural plasticity and recovery of consciousness in some patients with DOC. Our group showed that tDCS can effectively modulate the cortical excitability of patients with DOC, especially in patients with MCS (Bai et al., 2017a,c). Martens et al. found that 4 weeks of home-based tDCS moderately improved the recovery of signs of consciousness in patients with MCS (Martens et al., 2018). Similarly, Zhang and his colleagues reported 20 sessions of tDCS can improve CRS$\mathrm{R}$ scores and modulate the P300 amplitude in patients with MCS. The P300 has been commonly used to detect residual awareness in patients with DOC (Giacino et al., 2018). Several studies have also showed that patients with MCS can benefit from tDCS over the left dorsolateral prefrontal cortex (DLPFC) (Angelakis et al., 2014; Thibaut et al., 2014, 2015, 2017; Dimitri et al., 2017). Except the treatment effect of tDCS in patients with DOC, tDCS induced changes in cortical connectivity and excitability is useful in differentiating MCS from UWS patients (Naro et al., 2015). However, another study discovered that tDCS of the left DLPFC did not have remarkable clinical and EEG effects in patients with DOC (Estraneo et al., 2017). A critical problem of tDCS in patients with DOC is stimulated area. The left DLPFC is common in traumatic brain injury, Huang et al. (2017) chose the posterior parietal cortex as the site of stimulation. Researchers found $\mathrm{tDCS}$ of the posterior parietal cortex improves the recovery of clinical signs of consciousness in some patients with MCS (Huang et al., 2017). For researching the mechanism of change of tDCS in patients with DOC, a more focal stimulation is important. However, The main disadvantage of conventional tDCS is that it produces diffuse brain current flow. It is difficult to interpret whether produced effects are due to stimulation of the targeted cortical region or neighboring anatomical area (Bai et al., 2014; To et al., 2016).

High-Definition tDCS using the $4 \times 1$ smaller compact scalp electrodes, instead of the two large pad electrodes, is a new neuromodulation technique. HD-tDCS improves the spatial precision, resulting in focal neural and specific behavioral changes (Dmochowski et al., 2011; Villamar et al., 2013; Shekhawat and Vanneste, 2018). HD-tDCS has been previously reported to improve motor function, verbal learning, working memory, and pain and tinnitus control (Borckardt et al., 2012; Caparelli-Daquer et al., 2012; Kuo et al., 2013; Donnell et al., 2015; Nikolin et al., 2015; Shekhawat et al., 2016). It has been shown to reliably target specific brain areas and produce plastic changes that may outlast conventional tDCS (Kuo et al., 2013; Hogeveen et al., 2016). There is no study has examined the impact of HD-tDCS on DOC to date.
The CRS- $\mathrm{R}$ is a standardized behavioral assessment measure that has been widely used for diagnostic assessment and outcome measurement in patients with DOC (Gerrard et al., 2014). However, the rate of clinical misdiagnosis based on the CRS-R remains high (Xie et al., 2017). Previous results have demonstrated EEG can detect and analyze brain activity in clinical practice (Lehembre et al., 2012; Bai et al., 2017a,b). Our previous research has found the quantitative EEG was useful for assessment of the effect of tDCS and rTMS in patients with DOC (Bai et al., 2017a,c; Xia et al., 2017). EEG coherence has been applied to evaluate the effective connectivity of DOC, a high coherence hints at an increased functional interplay between the underlying neuronal networks (Rampil, 1998; Davey et al., 2000; Bai et al., 2017a,c).

We applied resting state EEG and CRS-R scores for assessing the effect in patients with DOC treated with long-lasting HDtDCS. We aim to confirm that HD-tDCS applied to the precuneus on patients with DOC could produce clinically useful behavioral modifications. We also want to find direct EEG evidence to demonstrate the efficacy of HD-tDCS in patients with DOC.

\section{MATERIALS AND METHODS}

\section{Patients}

We enrolled medically stable 18 patients with DOC hospitalized in Department of Neurosurgery, Zhengzhou Central Hospital Affiliated to Zhengzhou University, between October 2016 and June 2017. Due to pulmonary infection, phlebothrombosis, and other clinical interferences, 11 patients (5 VS and $6 \mathrm{MCS}$, mean age:52.8 years, range: $30.0-71.0$ years, 4 females, and 7 males) completed the entire experiment (Table 1). Inclusion criteria were VS/UWS or MCS patients, according to the JFK CRS-R scores. We excluded patients with DOC who had precuneus lesions, have had tDCS treatment before or last less than 3 months to avoid the spontaneous recovery period. Participants who had pacemakers, aneurysm clips, other devices implanted or other treatments and drugs which modifying cortical-excitability were also eliminated. The present study was approved by the ethics committee of the Zhengzhou Central Hospital Affiliated to Zhengzhou University.

\section{Design and HD-tDCS: Stimulation Protocol}

All patients received HD-tDCS modulation $(2 \mathrm{~mA}, 20 \mathrm{~min}$, anode centered over the precuneus) for two session per day over 14 consecutive days (Figure 1). The CRS-R assessments were conducted at four time points: before the experiment (T0), after a single session of HD-tDCS (T1), after the treatment of 7 days (T2), and 14 days (T3). In this study, any side effects of HD-tDCS were monitored and reported.

\section{HD-tDCS}

4 x1-Ring high-definition electrodes with an anode center electrode overlying the targeted brain area surrounded by four cathodal electrodes were used to deliver direct current to the 
TABLE 1 | Demographic details of the patients included in the study.

\begin{tabular}{|c|c|c|c|c|c|c|c|c|c|c|c|}
\hline \multirow[t]{2}{*}{ Patient } & \multirow[t]{2}{*}{ Age } & \multirow[t]{2}{*}{ Etiology } & \multirow[t]{2}{*}{ MRI findings } & \multirow[t]{2}{*}{$\begin{array}{l}\text { Duration } \\
\text { (months) }\end{array}$} & \multicolumn{7}{|c|}{ CRS-R } \\
\hline & & & & & A & $\mathbf{v}$ & $\mathbf{M}$ & OM & C & Ar & Total \\
\hline MCR1 & $66-70$ & Hemorrhage & Left frontal-parietal lesions, diffuse atrophy & 3 & 2 & 3 & 3 & 1 & 0 & 2 & 11 \\
\hline MCR2 & $51-55$ & Hemorrhage & Left frontal-temporal lesion & 3 & 2 & 2 & 2 & 1 & 0 & 1 & 8 \\
\hline MCR3 & $56-60$ & Hemorrhage & Left hemisphere lesion, diffuse cortical atrophy & 3 & 2 & 3 & 2 & 1 & 0 & 2 & 10 \\
\hline MCR4 & $26-30$ & Hemorrhage & Left parietal-temporal-thalamus lesion & 6 & 1 & 2 & 2 & 1 & 0 & 1 & 7 \\
\hline MCR5 & $71-75$ & Hemorrhage & Left frontal-temporal-parietal lesions & 4 & 2 & 2 & 3 & 1 & 0 & 2 & 10 \\
\hline MCR6 & $51-55$ & Hemorrhage & Right frontal-parietal-thalamus lesions & 3 & 1 & 2 & 2 & 1 & 0 & 2 & 8 \\
\hline VS1 & $36-40$ & Hemorrhage & Left frontal -temporal cortical atrophy & 6 & 1 & 1 & 2 & 0 & 0 & 2 & 6 \\
\hline VS2 & $61-65$ & Hemorrhage & Left hemisphere lesion & 3 & 1 & 1 & 2 & 1 & 0 & 1 & 6 \\
\hline VS3 & $51-55$ & Hemorrhage & Right frontal-temporal cortical atrophy & 3 & 1 & 1 & 2 & 1 & 0 & 1 & 6 \\
\hline VS4 & $36-40$ & $\mathrm{TBI}$ & Bilateral frontal and diffuse cortical atrophy. & 8 & 1 & 1 & 2 & 1 & 0 & 1 & 6 \\
\hline VS5 & $51-55$ & TBI & Left frontal-temporal, diffuse cortical atrophy & 6 & 1 & 0 & 2 & 0 & 0 & 1 & 4 \\
\hline
\end{tabular}

CRS-R, Coma Recovery Scale-Revised scores; A, auditory; V, visual; M, motor; OM, oromotor; C, communication; Ar, arousal.

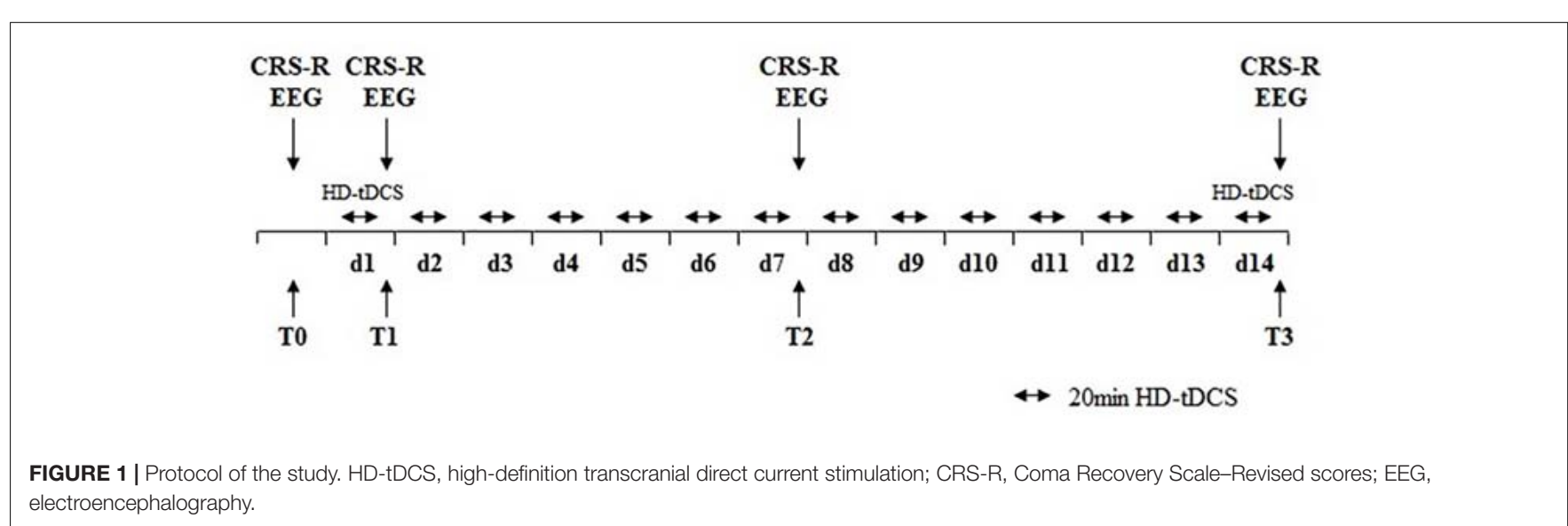

scalp with the application of $\mathrm{Ag} / \mathrm{AgCl}$ ring electrodes (Model 4x1-C2: Soterix Medical Inc., New York, NY, United States). HD-tDCS enables a more restricted cortical neuromodulation and leads to higher electric fields. Electrodes were held in place by specially designed plastic casings embedded in a 32-channel EEG recording cap. The center electrode (anode) was placed at $\mathrm{Pz}$ according to the international 10-20 EEG system, and four cathodal electrodes were placed approximately $3.5 \mathrm{~cm}$ radially from $\mathrm{Pz}$; corresponding roughly to locations $\mathrm{Cz}, \mathrm{P} 3, \mathrm{P} 4$, and $\mathrm{POz}$ (Figure 3A).

\section{EEG Recording and Pre-processing}

We used 32 EEG recorder (Nicolet EEG V32, Natus, United States). EEG recorded at four time points: before the experiment (T0), after a single session of HD-tDCS (T1), after the treatment of 7 days (T2), and 14 days (T3). EEG signals were continuously recorded from 32 channels at positions of the International 10/20 system. The electrodes with the setting of a band-pass filtered at DC to $1000 \mathrm{~Hz}$ in the recorder. The EEG signal was digitized at a sampling rate of $2.5 \mathrm{kHz}$. The skin impedance was maintained below $5 \mathrm{k} \Omega$. EEG recordings were carried out while patients were behaviorally awake.
Off-line analysis was carried out using EEGLAB 12.0.2.5b, running in a MATLAB environment (version 2013b, Math Works Inc., Natick, Massachusetts, United States). The 50-Hz power signal was removed by a notch filter. The independent component analysis function was used to identify and remove the artifact-relevant components. The EEG data were down-sampled to $500 \mathrm{~Hz}$ and average referenced. Then, the EEG date were divided into epochs of $10 \mathrm{~s}$ with $50 \%$ overlap in each patient.

\section{EEG Analysis}

Coherence was measured using spectral cross-correlation and normalized power spectra of signals obtained from two electrodes with the following equation:

$$
\operatorname{Coh}_{\mathrm{xy}}(f)=\frac{\left|P_{\mathrm{xy}}(f)\right|^{2}}{P_{\mathrm{xx}}(f) P_{\mathrm{yy}}(f)}
$$

where $P_{\mathrm{xy}}(f)$ was the cross-power spectral density and $P_{\mathrm{xx}}(f)$ and $P_{\mathrm{yy}}(f)$ were the respective auto-power spectral densities of the signals.

As shown in Figure 2A, the frontal region included electrodes Fp1, Fp2, Fz, F3, F4, Fc5, Fc6, Fc1, FC2, F7, and F8; the central 


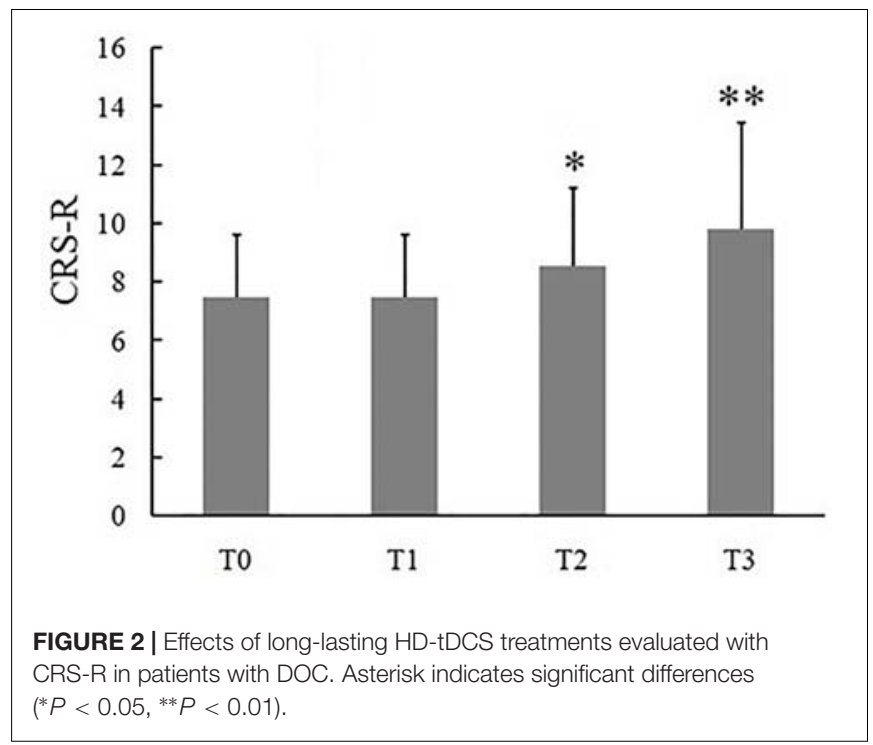

region included electrodes $\mathrm{CZ}, \mathrm{C} 3, \mathrm{C} 4, \mathrm{Cp} 1, \mathrm{Cp} 2, \mathrm{Cp} 5$, and $\mathrm{Cp} 6$; the parietal region included electrodes $\mathrm{Pz}, \mathrm{P} 3, \mathrm{P} 4$, and Poz. Centralparietal coherence was calculated using pairwise electrodes from the central and parietal regions. The frontal inter-hemisphere and central inter-hemisphere coherences were also calculated.

\section{Statistics}

The statistics were performed via SPSS for Windows, version 17.0. The Wicoxon signed-rank test was used to analyze the effects of HD-tDCS on CRS-R. And the Kolmogorov-Smirnov test was utilized to observe the coherence between different regions at the delta bands. Bonferroni correction was conducted after multiple comparisons. $P<0.05$ was regarded as statistically significant.

\section{RESULTS}

\section{Effects of the HD-tDCS Treatment as Measured by CRS-R}

Eleven (6 MCS and 5 VS) patients with chronic DOC completed the treatment, with no specific side effects, such as redness of the skin, signs of discomfort or epilepsy. 9/11 (72\%) patients (54\% of responders, $6 \mathrm{MCS}$ and $3 \mathrm{VS}$ ) showed the CRS-R scores increased after 14 days of stimulation (Table 2). The CRS-R scores increased with the treatment going on, compared with the baselines, and the CRS-R score at the 7 day was significantly higher than the baseline (Figure 2, $P<0.05$ ). It demonstrated that long-lasting HD-tDCS treatment can improve the recovery of consciousness in patients with DOC, whereas behavioral changes were not observed at just one session of stimulation.

\section{Effects of the HD-tDCS Treatment as Measured by EEG}

The coherence in the delta bands between the defined central and parietal regions was calculated (Figure 3B). Results showed that it decreased with the treatment going on, and the coherence
TABLE 2 | Clinical evaluation of the patients on day 7 and 14.

\begin{tabular}{|c|c|c|c|c|c|c|c|c|}
\hline \multirow[t]{2}{*}{ Patient } & \multicolumn{7}{|c|}{$\begin{array}{l}\text { CRS-R improvement } \\
\text { (day } 7 / 14 \text { ) }\end{array}$} & \multirow[t]{2}{*}{ Changes of diagnosis } \\
\hline & A & $\mathbf{v}$ & $\mathbf{M}$ & OM & C & Ar & Total & \\
\hline MCR1 & $0 / 1$ & $0 / 0$ & $0 / 0$ & $0 / 0$ & $0 / 0$ & $0 / 0$ & $0 / 1$ & MCS- elevated to MCS+ \\
\hline MCR2 & $0 / 0$ & $1 / 1$ & $1 / 1$ & $0 / 0$ & $0 / 0$ & $1 / 1$ & $3 / 3$ & Remained MCS- \\
\hline MCR3 & $0 / 1$ & $0 / 0$ & $1 / 2$ & $0 / 1$ & $0 / 1$ & $0 / 0$ & $1 / 5$ & MCS- elevated to MCS+ \\
\hline MCR4 & $0 / 2$ & $1 / 1$ & $0 / 1$ & $0 / 0$ & $0 / 1$ & $1 / 1$ & $2 / 6$ & MCS- elevated to MCS+ \\
\hline MCR5 & $0 / 0$ & $0 / 1$ & $0 / 0$ & $0 / 0$ & $0 / 0$ & $0 / 0$ & $0 / 1$ & Remained MCS- \\
\hline MCR6 & $1 / 2$ & $1 / 1$ & $1 / 1$ & $1 / 2$ & $0 / 0$ & $0 / 0$ & $4 / 6$ & MCS- elevated to MCS+ \\
\hline VS1 & $0 / 0$ & $0 / 0$ & $0 / 0$ & 0/0 & $0 / 0$ & $0 / 0$ & $0 / 0$ & Remained VS \\
\hline VS2 & 0/0 & $1 / 1$ & $0 / 0$ & $0 / 0$ & $0 / 0$ & $0 / 1$ & $1 / 2$ & Remained VS \\
\hline VS3 & $0 / 0$ & $0 / 1$ & $0 / 0$ & $0 / 0$ & $0 / 0$ & $0 / 0$ & $0 / 1$ & Remained VS \\
\hline VS4 & 0/0 & $0 / 0$ & $0 / 0$ & $0 / 0$ & $0 / 0$ & $1 / 1$ & $1 / 1$ & Remained VS \\
\hline VS5 & 0/0 & $0 / 0$ & $0 / 0$ & 0/0 & $0 / 0$ & $0 / 0$ & 0/0 & Remained VS \\
\hline
\end{tabular}

CRS-R, Coma Recovery Scale-Revised scores; A, auditory; V, visual; M, motor; OM, oromotor; C, communication; Ar, arousal.

index at T3 was higher significantly than that at T0 $(P<0.05)$. Figure 3C showed behavioral improvement was not discovered after the first stimulation. But the delta band coherence changed in the frontal inter -hemisphere regions in some patients with DOC. Besides, it reduced remarkably at day $7(P<0.05)$. Similarly, as shown in Figure 3D, the coherence between the central inter- hemisphere regions reduced after stimulation, and especially on day 7 and $14(P<0.05)$.

\section{DISCUSSION}

Several studies have reported effectiveness of conventional tDCS over the left DLPFC in patients with DOC (Angelakis et al., 2014; Thibaut et al., 2014, 2015, 2017; Dimitri et al., 2017). Patients with MCS but not VS are more easily benefit from tDCS at the left DLPFC. Recently, a sham-controlled randomized clinical trial investigated that 9/37 (27\%) patients with MCS showed improvements after conventional tDCS over the posterior parietal cortex (Huang et al., 2017). A randomized double-blinded sham-controlled cross-over study didn't support effectiveness of conventional tDCS over the left DLPFC in patients with DOC (Estraneo et al., 2017). Estraneo et al.'s (2017) study didn't observe relevant behavioral and EEG changes in the single or repeated stimulation over the left DLPFC. What's more, owing to low spatial resolution of conventional tDCS, it was difficult to explain causality between stimulation of target brain region and the behavioral changes (Kuo et al., 2013). In addition, many studies only used CRS-R to evaluate treatment effects of conventional tDCS. In fact behavioral changes are not always observed in patients with DOC, particularly in short-term modulation. The precuneus as known to be involved in conscious processes plays an important role in consciousness recovery (Laureys and Schiff, 2012). Therefore, we targeted anodal HDtDCS at the precuneus to evaluated modulation clinical effects and EEG oscillation in patients with DOC. We found that long-lasting HD-tDCS improved the recovery of consciousness 


\section{A}

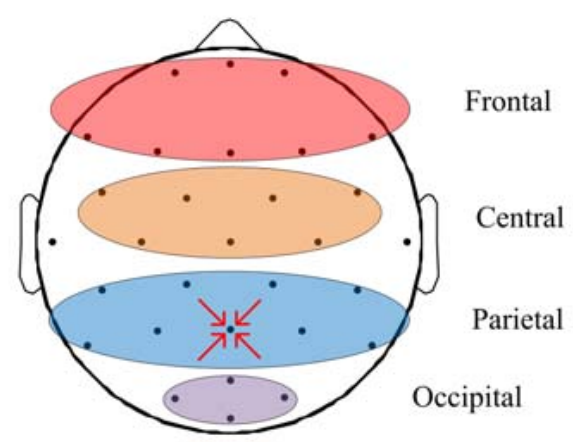

C

Frontal inter-hemisphere

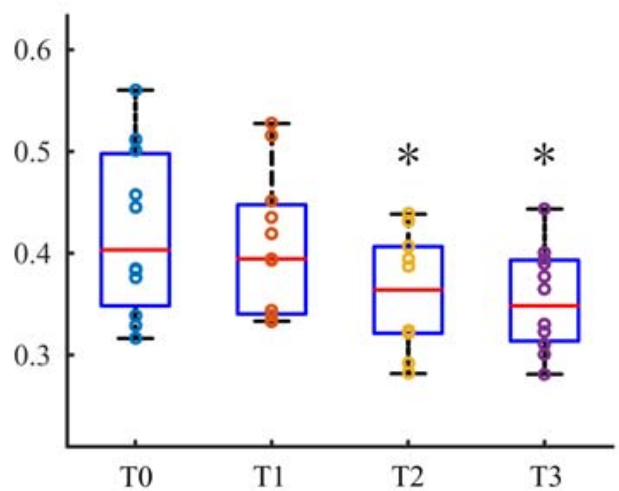

B

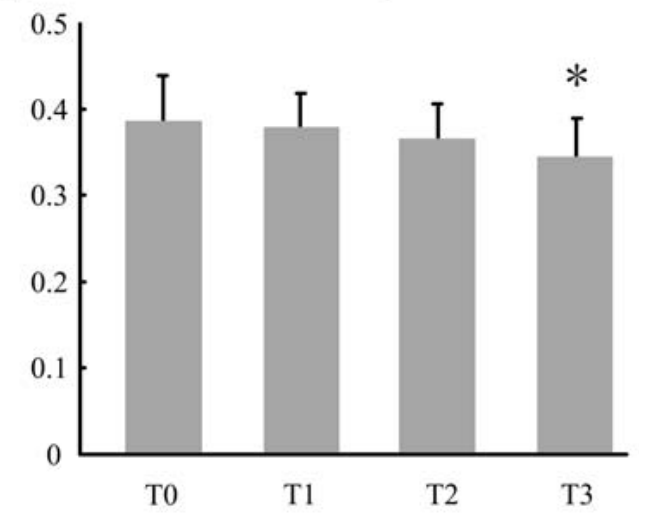

D

Central inter-hemisphere

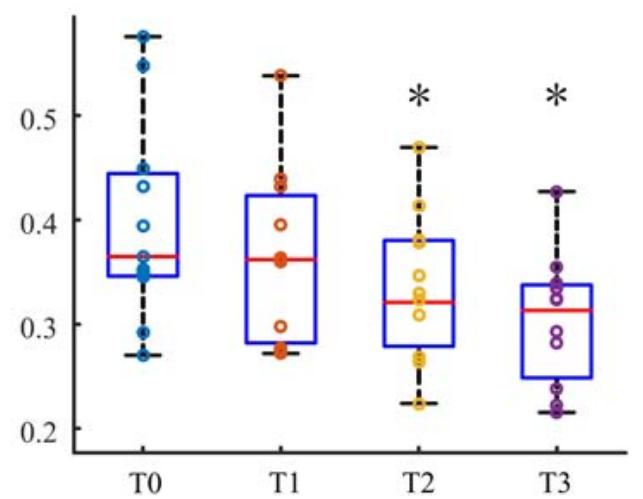

FIGURE 3 | The coherence between different regions in the delta bands. (A) Defined different regions of brain. (B) The coherence between central and parietal regions. (C) The frontal inter-hemisphere coherence. (D) The central inter-hemisphere coherence. Asterisks indicate statistically significant differences ( $P<0.05)$.

in patients with MCS and some patients in VS. Resting state EEG showed a significant reduction of the coherence between the central and parietal region at the delta band. Significant decreasing of coherence was also found at inter-hemisphere of frontal and central. These changes occurred in different time windows and brain regions for patients with DOC. No side effects such as discomfort, skin burn, and seizures were observed after any of the stimulation.

High-Definition tDCS delivery system was developed to enhance the spatial accuracy of tDCS, which is believed to enhance the clinical effects of this therapeutic tool. HD-tDCS uses the $4 \times 1$ montage of stimulating electrodes, which generates in maximal focused electric field strength under the target electrode with brain current flow constrained by the ring radius (Kuo et al., 2013; Gbadeyan et al., 2016; Hogeveen et al., 2016). Thus, it produced more spatially restricted electric field, as compared to the conventional electrode placement. HD-tDCS has the characteristics of high spatial resolution and more focused electric field than conventional tDCS protocols, which may offer the opportunity to explore the contribution of stimulation cortical target to consciousness. The efficacy of HD-tDCS for investigating motor cortex excitability, conscious movement intention, fibromyalgia, pain, tinnitus, verbal learning, and memory functioning have been reported (Borckardt et al., 2012; Caparelli-Daquer et al., 2012; Kuo et al., 2013; Donnell et al., 2015; Nikolin et al., 2015; Shekhawat et al., 2016). To our knowledge, there is no study has examined the impact of HDtDCS on DOC. Targeting the precuneus using HD-tDCS will help probably to understand the recovery mechanisms of clinical sign of consciousness better.

The site of stimulation is also a critical scientific issue (Xia et al., 2017). The left DLPFC, cerebellum and the posterior parietal cortex were selected as the stimulation sites in DOC frequently. Cerebellum involves in short- and longterm habituation of unconditioned responses (Naro et al., 2016; Bocci et al., 2018), but it is not essential for consciousness. Cerebellar tDCS may be useful for ameliorating the level of consciousness (Naro et al., 2016). Both Left DLPFC and the precuneus are involved in conscious processes (Schiff, 2010; Xia et al., 2017). Conventional tDCS of the DLPFC have shown promising results in patients with MCS, which requires gray matter integrity (Thibaut et al., 2015). The probability of damage in DLPFC is higher than the posterior parietal cortex in DOC, the latter seems a better stimulation site in clinic (Pandya and Seltzer, 1982). The precuneus is associated with memory retrieval, controlling spatial aspects of behavior and Visual-spatial 
visualization (Wenderoth et al., 2005; Ionta et al., 2014; Blanke et al., 2015; Kragel and Polyn, 2016; Rissman et al., 2016). The precuneus plays an important role in the mesocircuit model. Thibaut et al. (2015) found the precuneus metabolism and behavioral level supporting the fronto-parietal network correlate with outcomes in DOC (Thibaut et al., 2015). Moreover, the precuneus seems to be a brain region that can differentiate patients with MCS from VS.

As mentioned in the introduction, a single session of tDCS over the left DLPF transiently improves CRS-R total scores in patients with MCS (Giacino et al., 2012). Results of another study suggest that repeated tDCS improves the recovery of consciousness in 56\% patients with DOC (Thibaut et al., 2017). In this study, We didn't observe any patient showed behavioral response to $\mathrm{HD}$-tDCS after the first session of stimulation. Interestingly, we observed that $6 / 11$ patients $(54 \%$ of responders, 4 MCS and 2 VS) showed the CRS-R scores significant improvement after 7 days of stimulation. The improvement was observed in 6 patients with MCS and 3 patients with VS after 14 days of stimulation. Four patients with MCS - rose from MCS - to MCS +. These results suggested that repeated HDtDCS daily could promote consciousness level in MCS, whereas all VS remained previous consciousness state. These results suggested that the first session is not predictive of a future positive effect of the stimulation on the level of consciousness. Stimulation term is another critical issue, long-lasting stimulation possible improves neuroplasticity and strengthen the effect of the stimulation. In the future, longer-term stimulations (such as 20,30, or 60 days) should be considered to discover the potential of recovery effects in patients with DOC. MCS patients have more prominent potential of neural plasticity, which attain more benefit from HD-tDCS.

To reveal the mechanism of action and clinical effects of HD-tDCS over the precuneus of patients with DOC, functional connectivity of coherence was investigated using based resting state EEG. Previous studies have shown that the severity of DOC was correlated with increased low-frequency band power and decreased high-frequency band power (Bai et al., 2017a,b,c; Xia et al., 2017). Our data showed the significant reduction of shortrange central-parietal coherence at the delta band after longlasting HD-tDCS modulation. The long-range frontal-parietal coherence in the delta band did not decrease. But the frontal inter-hemisphere coherence significantly decreased in the delta band with increasing stimulation sessions. Besides, remarkably decrease compared to baseline was first shown at day 7. Similar results were observed in the central inter-hemisphere. Behavioral improvement was not discovered after the first stimulation. But the delta band coherence changes in brain implied a cortical response to the stimulation. Some patients who had no response to the first stimulation CRS-R scores improved after the whole stimulation session. These indicated that HD-tDCS could effectively alter the brain electrical activity. Accordingly, HD-tDCS induced variation of delta band coherence negatively correlated with the patients' CRS-R scores to some extent.

Studies demonstrated that the degree of DOC may be correlated with increased low-frequency band power in EEG patients (Bai et al., 2017a,b,c; Xia et al., 2017). The alteration of delta oscillations is accompanied by function alterations in the brain (Cavinato et al., 2015). The rationale for performing HDtDCS in consciousness recovery remains unclear, but we found the trend of changes in the delta band in the frontal central and parietal regions. These changes can be summarized in a modulation of cortical coherence in short-range central-parietal and long-range frontal-parietal areas within a delta frequency range. Therefore, we considered that the changes occurring in the delta bands may provide evidence for supporting the modulating effects of HD-tDCS in patients with DOC.

Our study has several limitations. Firstly, the sample size was small. In the following research, we need recruit more patients with DOC to confirm and validate tDCS effectiveness. Secondly, this study lacked a randomized cross-over design and follow-up assessment, for long-term effect needs to be verified to determine its clinical effect. Thirdly, initial level of consciousness varied from VS/UWS to MCS. Therefore, future clinical trials should set up MCS and VS groups based on larger samples. In addition, we did not utilize neurophysiological and neuroimaging technology (e.g., event-related potential ERP, mainly the P300 component, functional MR), which would allow to better understand the treatment effects and mechanisms of HD-tDCS in patients with DOC (Zhang et al., 2017; Ragazzoni et al., 2019). What's more, P300 recording reflecting residual levels of awareness can assist in prognostication regarding 12-month recovery of consciousness for patients with DOC (Giacino et al., 2018). In future study, Multi-Modality technology should be applied together to provide a broader and more holistic evaluation of therapeutic efficacy.

\section{CONCLUSION}

In this study, we found that Long-lasting HD-tDCS over the precuneus could improve the recovery of consciousness in patients with DOC. EEG changes in the delta band were observed in fronta-central-parietal cortex, which provides direct evidence of the HD-tDCS protocol effects on the patients with DOC. Further studies are needed to verify the clinical effect of HD-tDCS on larger numbers of patients and expound the mechanism of the recovery of consciousness of HD-tDCS protocol.

\section{DATA AVAILABILITY}

All datasets generated for this study are included in the manuscript and/or the supplementary files.

\section{ETHICS STATEMENT}

This study was carried out in accordance with the recommendations of the ethics committee of the Zhengzhou Central Hospital with written informed consent from all subjects. All subjects gave written informed consent in accordance with the Declaration of Helsinki. The protocol was approved by the ethics committee of the Zhengzhou Central Hospital. 


\section{AUTHOR CONTRIBUTIONS}

YWD, JH, CL, and HZ designed the study. YG, JL, and XW collected the data. YB, XX, and YYD analyzed the data. $\mathrm{YB}$ and $\mathrm{CL}$ created the figures and tables. YG wrote and edited the manuscript.

\section{REFERENCES}

Angelakis, E., Liouta, E., Andreadis, N., Korfias, S., Ktonas, P., Stranjalis, G., et al. (2014). Transcranial direct current stimulation effects in disorders of consciousness. Arch. Phys. Med. Rehabil. 95, 283-289. doi: 10.1016/j.apmr.2013. 09.002

Bai, S., Dokos, S., Ho, K. A., and Loo, C. (2014). A computational modelling study of transcranial direct current stimulation montages used in depression. Neuroimage 87, 332-344. doi: 10.1016/j.neuroimage.2013.11.015

Bai, Y., Xia, X., Kang, J., Yang, Y., He, J., and Li, X. (2017a). TDCS modulates cortical excitability in patients with disorders of consciousness. Neuroimage Clin. 15, 702-709. doi: 10.1016/j.nicl.2017.01.025

Bai, Y., Xia, X., Li, X., Wang, Y., Yang, Y., Liu, Y., et al. (2017b). Spinal cord stimulation modulates frontal delta and gamma in patients of minimally consciousness state. Neuroscience 346, 247-254. doi: 10.1016/j.neuroscience. 2017.01.036

Bai, Y., Xia, X., Wang, Y., Guo, Y., Yang, Y., He, J., et al. (2017c). Frontoparietal coherence response to tDCS modulation in patients with disorders of consciousness. Int. J. Neurosci. 128, 587-594. doi: 10.1080/00207454.2017. 1403440

Blanke, O., Slater, M., and Serino, A. (2015). Behavioral, neural, and computational principles of bodily self-consciousness. Neuron 88, 145-166. doi: 10.1016/j. neuron.2015.09.029

Bocci, T., Ferrucci, R., Barloscio, D., Parenti, L., Cortese, F., Priori, A., et al. (2018). Cerebellar direct current stimulation modulates hand blink reflex: implications for defensive behavior in humans. Physiol. Rep. 6:e13471. doi: 10.14814/phy2. 13471

Borckardt, J. J., Bikson, M., Frohman, H., Reeves, S. T., Datta, A., Bansal, V., et al. (2012). A pilot study of the tolerability and effects of high-definition transcranial direct current stimulation (HD-tDCS) on pain perception. J. Pain 13, 112-120. doi: 10.1016/j.jpain.2011.07.001

Bruno, M. A., Majerus, S., Boly, M., Vanhaudenhuyse, A., Schnakers, C., Gosseries, O., et al. (2012). Functional neuroanatomy underlying the clinical subcategorization of minimally conscious state patients. J. Neurol. 259, 1087 1098. doi: 10.1007/s00415-011-6303-7

Caparelli-Daquer, E. M., Zimmermann, T. J., Mooshagian, E., Parra, L. C., Rice, J. K., Datta, A., et al. (2012). "A pilot study on effects of $4 \mathrm{x} 1$ high-definition tDCS on motor cortex excitability," in Proceedings of the Annual International Conference of the IEEE Engineering in Medicine and Biology Society, (San Diego, CA: IEEE), 735-738.

Cavinato, M., Genna, C., Manganotti, P., Formaggio, E., Storti, S. F., Campostrini, S., et al. (2015). Coherence and consciousness: study of fronto-parietal gamma synchrony in patients with disorders of consciousness. Brain Topogr. 28, 570579. doi: 10.1007/s10548-014-0383-5

Cossu, G. (2014). Therapeutic options to enhance coma arousal after traumatic brain injury: state of the art of current treatments to improve coma recovery. Br. J. Neurosurg. 28, 187-198. doi: 10.3109/02688697.2013.841845

Davey, M. P., Victor, J. D., and Schiff, N. D. (2000). Power spectra and coherence in the EEG of a vegetative patient with severe asymmetric brain damage. Clin. Neurophysiol. 111, 1949-1954.

Della Pepa, G. M., Fukaya, C., La Rocca, G., Zhong, J., and Visocchi, M. (2013). Neuromodulation of vegetative state through spinal cord stimulation: where are we now and where are we going? Stereotact. Funct. Neurosurg. 91, 275-287. doi: $10.1159 / 000348271$

Dimitri, D., De Filippis, D., Galetto, V., and Zettin, M. (2017). Evaluation of the effectiveness of transcranial direct current stimulation (tDCS) and psychosensory stimulation through DOCS scale in a minimally conscious subject. Neurocase 23, 96-104. doi: 10.1080/13554794.2017.130 5112

\section{FUNDING}

This research was supported by the Key Scientific Research Projects of Henan Province Universities and Colleges (19A320041 and 18B310037) and the Medicine and Health Science and Technology Project of Zhejiang Province (2019RC254).

Dmochowski, J. P., Datta, A., Bikson, M., Su, Y., and Parra, L. C. (2011). Optimized multi-electrode stimulation increases focality and intensity at target. J. Neural. Eng. 8:046011. doi: 10.1088/1741-2560/8/4/046011

Donnell, A. D., Nasciment, T., Lawrence, M., Gupta, V., Zieba, T., Truong, D. Q., et al. (2015). High-definition and non-invasive brain modulation of pain and motor dysfunction in chronic TMD. Brain Stimul. 8, 1085-1092. doi: 10.1016/j. brs.2015.06.008

Estraneo, A., Pascarella, A., Moretta, P., Masotta, O., Fiorenza, S., Chirico, G., et al. (2017). Repeated transcranial direct current stimulation in prolonged disorders of consciousness: a double-blind cross-over study. J. Neurol. Sci. 375, 464-470. doi: 10.1016/j.jns.2017.02.036

Gbadeyan, O., McMahon, K., Steinhauser, M., and Meinzer, M. (2016). Stimulation of dorsolateral prefrontal cortex enhances adaptive cognitive control: a highdefinition transcranial direct current stimulation study. J. Neurosci. 36, 1253012536. doi: 10.1523/JNEUROSCI.2450-16.2016

Gerrard, P., Zafonte, R., and Giacino, J. T. (2014). Coma recovery scale-revised: evidentiary support for hierarchical grading of level of consciousness. Arch. Phys. Med. Rehabil. 95, 2335-2341. doi: 10.1016/j.apmr.2014.06.018

Giacino, J. T., Fins, J. J., Laureys, S., and Schiff, N. D. (2014). Disorders of consciousness after acquired brain injury: the state of the science. Nat. Rev. Neurol. 10, 99-114. doi: 10.1038/nrneurol.2013.279

Giacino, J. T., Katz, D. I., Schiff, N. D., Whyte, J., Ashman, E. J., Ashwal, S., et al. (2018). Practice guideline update recommendations summary: disorders of consciousness: report of the guideline development, dissemination, and implementation subcommittee of the american academy of neurology; the american congress of rehabilitation medicine; and the national institute on disability, independent living, and rehabilitation research. Neurology 91, 450460. doi: 10.1212/wnl.0000000000005926

Giacino, J. T., Whyte, J., Bagiella, E., Kalmar, K., Childs, N., Khademi, A., et al. (2012). Placebo-controlled trial of amantadine for severe traumatic brain injury. N. Engl. J. Med. 366, 819-826. doi: 10.1056/NEJMoa1102609

Hogeveen, J., Grafman, J., Aboseria, M., David, A., Bikson, M., and Hauner, K. K. (2016). Effects of high-definition and conventional tDCS on response inhibition. Brain Stimul. 9, 720-729. doi: 10.1016/j.brs.2016.04.015

Huang, W., Wannez, S., Fregni, F., Hu, X., Jing, S., Martens, G., et al. (2017) Repeated stimulation of the posterior parietal cortex in patients in minimally conscious state: a sham-controlled randomized clinical trial. Brain Stimul. 10, 718-720.

Ionta, S., Martuzzi, R., Salomon, R., and Blanke, O. (2014). The brain network reflecting bodily self-consciousness: a functional connectivity study. Soc. Cogn. Affect. Neurosci. 9, 1904-1913. doi: 10.1093/scan/nst185

Kragel, J. E., and Polyn, S. M. (2016). Decoding episodic retrieval processes: frontoparietal and medial temporal lobe contributions to free recall. J. Cogn. Neurosci. 28, 125-139. doi: 10.1162/jocn_a_00881

Kuo, H. I., Bikson, M., Datta, A., Minhas, P., Paulus, W., Kuo, M. F., et al. (2013). Comparing cortical plasticity induced by conventional and high-definition $4 \mathrm{x}$ 1 ring tDCS: a neurophysiological study. Brain Stimul. 6, 644-648. doi: 10.1016/ j.brs.2012.09.010

Laureys, S., and Schiff, N. D. (2012). Coma and consciousness: paradigms (re) framed by neuroimaging. Neuroimage 61, 478-491. doi: 10.1016/j.neuroimage. 2011.12.041

Lefaucheur, J. P., Antal, A., Ayache, S. S., Benninger, D. H., Brunelin, J., Cogiamanian, F., et al. (2017). Evidence-based guidelines on the therapeutic use of transcranial direct current stimulation (tDCS). Clin. Neurophysiol. 128, 56-92. doi: 10.1016/j.clinph.2016.10.087

Lehembre, R., Marie-Aurelie, B., Vanhaudenhuyse, A., Chatelle, C., Cologan, V. Leclercq, Y., et al. (2012). Resting-state EEG study of comatose patients: a connectivity and frequency analysis to find differences between vegetative and minimally conscious states. Funct. Neurol. 27, 41-47. 
Martens, G., Lejeune, N., O’Brien, A. T., Fregni, F., Martial, C., Wannez, S., et al. (2018). Randomized controlled trial of home-based 4-week tDCS in chronic minimally conscious state. Brain Stimul. 11, 982-990. doi: 10.1016/j.brs.2018. 04.021

Naro, A., Calabro, R. S., Russo, M., Leo, A., Pollicino, P., Quartarone, A., et al. (2015). Can transcranial direct current stimulation be useful in differentiating unresponsive wakefulness syndrome from minimally conscious state patients? Restor. Neurol. Neurosci. 33, 159-176. doi: 10.3233/rnn- 140448

Naro, A., Russo, M., Leo, A., Cannavo, A., Manuli, A., Bramanti, A., et al. (2016). Cortical connectivity modulation induced by cerebellar oscillatory transcranial direct current stimulation in patients with chronic disorders of consciousness: a marker of covert cognition? Clin. Neurophysiol. 127, 1845-1854. doi: 10.1016/ j.clinph.2015.12.010

Nikolin, S., Loo, C. K., Bai, S., Dokos, S., and Martin, D. M. (2015). Focalised stimulation using high definition transcranial direct current stimulation (HD-tDCS) to investigate declarative verbal learning and memory functioning. Neuroimage 117, 11-19. doi: 10.1016/j.neuroimage.2015. 05.019

Pandya, D. N., and Seltzer, B. (1982). Intrinsic connections and architectonics of posterior parietal cortex in the rhesus monkey. J. Comp. Neurol. 204, 196-210. doi: 10.1002/cne.902040208

Ragazzoni, A., Di Russo, F., Fabbri, S., Pesaresi, I., Di Rollo, A., Perri, R. L., et al. (2019). "Hit the missing stimulus". A simultaneous EEG-fMRI study to localize the generators of endogenous ERPs in an omitted target paradigm. Sci. Rep. 9:3684. doi: 10.1038/s41598-019-39812-z

Rampil, I. J. (1998). A primer for EEG signal processing in anesthesia. Anesthesiology 89, 980-1002.

Rissman, J., Chow, T. E., Reggente, N., and Wagner, A. D. (2016). Decoding fMRI signatures of real-world autobiographical memory retrieval. J. Cogn. Neurosci. 28,604-620. doi: 10.1162/jocn_a_00920

Schiff, N. D. (2010). Recovery of consciousness after brain injury: a mesocircuit hypothesis. Trends Neurosci. 33, 1-9. doi: 10.1016/j.tins.2009.11.002

Schiff, N. D., Giacino, J. T., Kalmar, K., Victor, J. D., Baker, K., Gerber, M., et al. (2007). Behavioural improvements with thalamic stimulation after severe traumatic brain injury. Nature 448, 600-603.

Shekhawat, G. S., Sundram, F., Bikson, M., Truong, D., De Ridder, D., Stinear, C. M., et al. (2016). Intensity, duration, and location of high-definition transcranial direct current stimulation for tinnitus relief. Neurorehabil. Neural Repair 30, 349-359. doi: 10.1177/1545968315595286

Shekhawat, G. S., and Vanneste, S. (2018). High-definition transcranial direct current stimulation of the dorsolateral prefrontal cortex for tinnitus modulation: a preliminary trial. J. Neural Transm. 125, 163-171. doi: 10.1007/ s00702-017-1808-6

Thibaut, A., Bruno, M. A., Ledoux, D., Demertzi, A., and Laureys, S. (2014). tDCS in patients with disorders of consciousness: sham-controlled randomized double-blind study. Neurology 82, 1112-1118. doi: 10.1212/wnl. 0000000000000260

Thibaut, A., Di Perri, C., Chatelle, C., Bruno, M. A., Bahri, M. A., Wannez, S., et al. (2015). Clinical response to tDCS depends on residual brain metabolism and grey matter integrity in patients with minimally conscious state. Brain Stimul. 8, 1116-1123. doi: 10.1016/j.brs.2015.07.024
Thibaut, A., Wannez, S., Donneau, A. F., Chatelle, C., Gosseries, O., Bruno, M. A., et al. (2017). Controlled clinical trial of repeated prefrontal tDCS in patients with chronic minimally conscious state. Brain Inj 31, 466-474. doi: 10.1080/ 02699052.2016.1274776

To, W. T., Hart, J., De Ridder, D., and Vanneste, S. (2016). Considering the influence of stimulation parameters on the effect of conventional and highdefinition transcranial direct current stimulation. Expert Rev. Med. Devices 13, 391-404. doi: 10.1586/17434440.2016.1153968

Tucker, C., and Sandhu, K. (2016). The effectiveness of Zolpidem for the treatment of disorders of consciousness. Neurocrit. Care 24, 488-493. doi: 10.1007/ s12028-015-0227-5

Villamar, M. F., Volz, M. S., Bikson, M., Datta, A., Dasilva, A. F., and Fregni, F. (2013). Technique and considerations in the use of $4 \times 1$ ring high-definition transcranial direct current stimulation (HD-tDCS). J. Vis. Exp. 77:e50309. doi: $10.3791 / 50309$

Wenderoth, N., Debaere, F., Sunaert, S., and Swinnen, S. P. (2005). The role of anterior cingulate cortex and precuneus in the coordination of motor behaviour. Eur. J. Neurosci. 22, 235-246. doi: 10.1111/j.1460-9568.2005.04176.x

Xia, X., Liu, Y., Bai, Y., Liu, Z., Yang, Y., Guo, Y., et al. (2017). Long-lasting repetitive transcranial magnetic stimulation modulates electroencephalography oscillation in patients with disorders of consciousness. Neuroreport 28, $1022-$ 1029. doi: 10.1097/wnr.0000000000000886

Xie, Q., Ni, X., Yu, R., Li, Y., and Huang, R. (2017). Chronic disorders of consciousness. Exp. Ther. Med. 14, 1277-1283. doi: 10.3892/etm.2017.4639

Yamamoto, T., Katayama, Y., Obuchi, T., Kobayashi, K., Oshima, H., and Fukaya, C. (2013). Deep brain stimulation and spinal cord stimulation for vegetative state and minimally conscious state. World Neurosurg. 80, S30.e1-S30.e9. doi: 10.1016/j.wneu.2012.04.010

Zhang, Y., and Song, W. (2018). Transcranial direct current stimulation in disorders of consciousness: a review. Int. J. Neurosci. 128, 255-261. doi: 10.1080/ 00207454.2017.1381094

Zhang, Y., Song, W., Du, J., Huo, S., Shan, G., and Li, R. (2017). Transcranial direct current stimulation in patients with prolonged disorders of consciousness: combined behavioral and event-related potential evidence. Front. Neurol. 8:620. doi: $10.3389 /$ fneur.2017.00620

Zhao, H., Qiao, L., Fan, D., Zhang, S., Turel, O., Li, Y., et al. (2017). Modulation of brain activity with noninvasive transcranial direct current stimulation (tDCS): clinical applications and safety concerns. Front. Psychol. 8:685. doi: 10.3389/ fpsyg.2017.00685

Conflict of Interest Statement: The authors declare that the research was conducted in the absence of any commercial or financial relationships that could be construed as a potential conflict of interest.

Copyright (c) 2019 Guo, Bai, Xia, Li, Wang, Dai, Dang, He, Liu and Zhang. This is an open-access article distributed under the terms of the Creative Commons Attribution License (CC BY). The use, distribution or reproduction in other forums is permitted, provided the original author(s) and the copyright owner(s) are credited and that the original publication in this journal is cited, in accordance with accepted academic practice. No use, distribution or reproduction is permitted which does not comply with these terms. 